\title{
The performance of the CMS ECAL Preshower detector at LHC
}

\section{Zong-Kai Liu'}

Department of Physics, National Central University

300 Jhongda Road, Jhongli, Taoyuan County 320, Taiwan

E-mail: Zong-Kai.Liulcern.ch

\section{Chia-Ming Kuo}

Department of Physics, National Central University

300 Jhongda Road, Jhongli, Taoyuan County 320, Taiwan

E-mail: Chia-Ming.Kuo@cern.ch

\section{Syue-Wei Li}

Department of Physics, National Central University

300 Jhongda Road, Jhongli, Taoyuan County 320, Taiwan

E-mail: Syue-Wei.Li@cern.ch

On behalf of the CMS collaboration

The Preshower detector is part of the CMS endcap electromagnetic calorimeter, located in front of the lead tungstate crystals. It comprises two orthogonal planes of silicon strip sensors each preceded by a thin layer of lead. The purpose of the Preshower is to identify two closely spaced photons from $\pi^{0}$ decays, allowing a reduction of one of the backgrounds to the two-photon decay channel of the intermediate mass Higgs boson $-\pi^{0} \mathrm{~s}$ in jets faking photons. Following a brief overview of the design of the Preshower, we present occupancy measurements and first insitu absolute calibration.

\footnotetext{
$1 \quad$ Speaker
} 


\section{Introduction}

The CMS [1] Preshower detector (ES) [2] is part of the CMS electromagnetic calorimeter (ECAL), located in front of the lead tungstate crystals in the endcaps. The coverage of the ES is $1.653<|\eta|<2.6$. The primary function of the ES is to identify two closely spaced photons from $\pi^{0}$ decays, allowing them to be distinguished from single photons that may come from decays of the intermediate mass Higgs boson (i.e. $\mathrm{H} \rightarrow \gamma \gamma$ ). The ES comprises 4 planes of silicon strip sensors (two planes per endcap) and associated mechanics, cooling and front end electronics. The sensors have an active area of $61 \times 61 \mathrm{~mm}^{2}$, divided into 32 strips. Following installation in Spring 2009, the ES has been fully operational throughout LHC running. There are in total 4288 sensors and thus 137216 strips. The total percentage of fully-functional strips is $99.8 \%$. The ES commissing and first results with $7 \mathrm{TeV}$ data can be found in [3].

\section{Preshower Occupancy in 7 TeVcollisions}

The ES occupancy is calculated as the percentage of strips with a reconstructed energy greater than $4 \sigma$ of the noise. Figure 1 shows the variation of occupancy with $\eta$ and $\Phi$, both compared to simulation, where absolute values of the MC occupancies have been normalized to the data. The $\Phi$ variations are due to the X-Y geometry of the ES, and are well-modeled by simulation. The occupancy increases as a function of $\eta$ as expected.
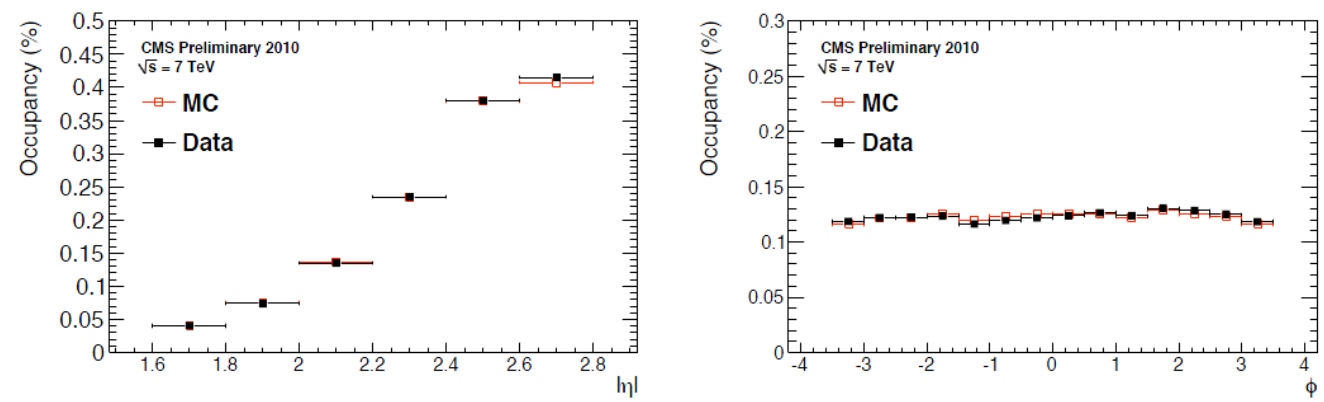

Figure 1: ES occupancy as functions of $\eta$ (left, averaged over all $\Phi$ ) and $\Phi$ (right, averaged over all $\eta)$.

\section{Preshower calibration and clustering}

The ES is a sampling calorimeter and essentially counts the number of charged particles passing through the silicon. The particles used to calibrate the ES are currently charged pions, with $\mathrm{p}>1 \mathrm{GeV} / \mathrm{c}$ and an average momentum of $6.1 \mathrm{GeV} / \mathrm{c}$. This means that they are nearly minimum ionizing, so for simplicity we refer to them as "MIPs".

Following a laboratory pre-calibration with cosmic rays (with $\sim 2.5 \%$ precision) we use charged pions from $7 \mathrm{TeV}$ collisions, with $\mathrm{p}>1 \mathrm{GeV} / \mathrm{c}$ pointing to the ES, to carry out the first in-situ ES MIP calibration. The MIP value is defined as the fitted peak position from the energy distribution. The observed S/N ratio for single ionizing particles, as shown in Fig 2 (left), is between 9 and 11. Figure 2 (right) shows the correlation between sensor MIP values obtained from in-situ calibration and the laboratory pre-calibration. The precision of the in-situ calibration is estimated to be about $2.2 \%$. 

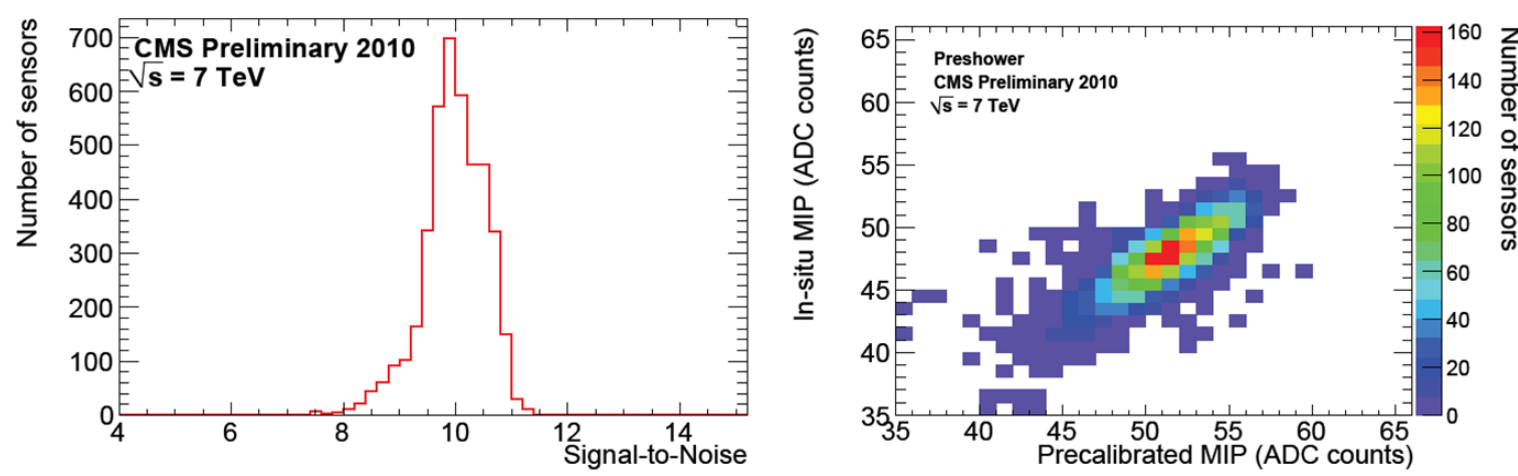

Figure 2: The ES signal to noise ratio for single particles (left) and the correlation between sensor MIP values obtained from in-situ calibration and pre-calibration (right).

In the endcap ECAL (EE), clusters of crystals are formed around seed crystals having $\mathrm{E}_{\mathrm{T}}>1 \mathrm{GeV}$. Then, EE cluster positions are extrapolated to the ES and ES clusters built. Figure 3 shows the total cluster energies measured by each plane of the ES for both data and simulation as well as the ratio of energies in the two ES planes. The bins at high MIP/ratio values are due to some saturation at high energies beacuse the ES was, for 2010, operated in a mode with a limited dynamic range but high efficiency for MIPs.
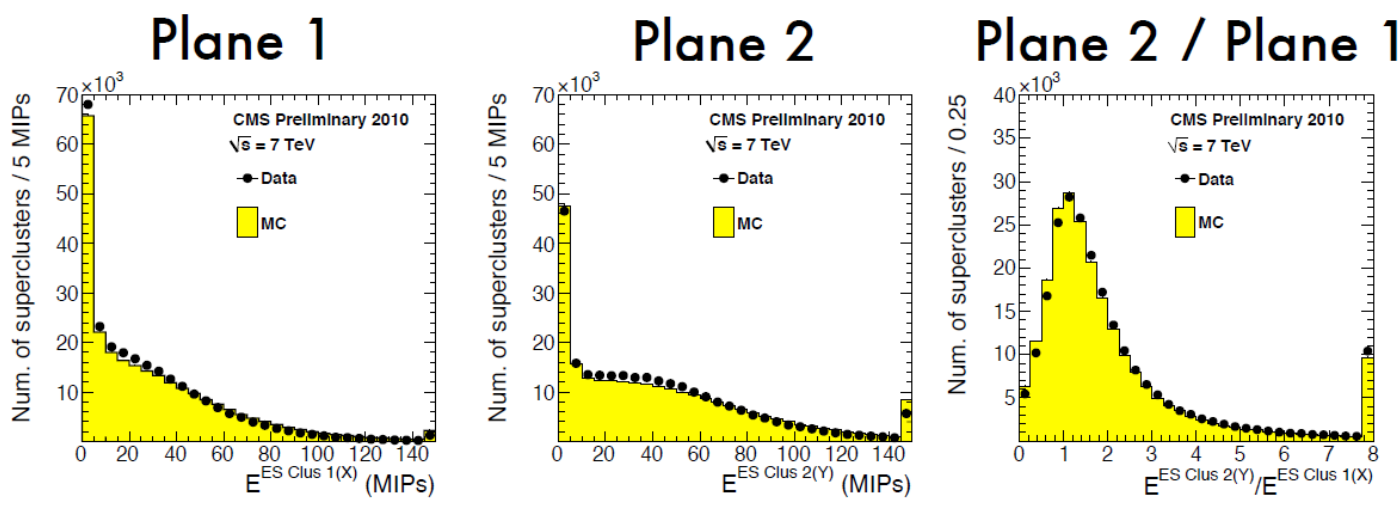

Figure 3: Comparison between data and simulation for the total cluster energies measured by each plane of the ES, expressed in units of MIP, as well as the ratio between the energies.

\section{Summary}

The ES has been operating stably throughout the 2009 and 2010 LHC running, with more than $99.8 \%$ of its strips fully functional. The first results compare well with simulation and are as expected.

\section{References}

[1] CMS Collaboration, The CMS experiment at the CERN LHC, JINST 0803 (2008) S08004.

[2] CMS Collaboration, CMS Physics TDR: Volume I (PTDR1), Detector Performance and Software, CERN/LHCC-2006-001 (2006).

[3] CMS Collaboration, Electromagnetic calorimeter commissioning and first results with 7 TeVdata, CMS NOTE -2010/012 (2010). 